\title{
San Diego county Nanopore SARS-Cov2 sequencing data shows metagenomic Prevotella, Haemophilus parainfluenzae, a lot of unknown species and chimeric reads
}

Sandeep Chakraborty

\section{Letter}

Prevotella was definitely a dominant factor in the the SARS-Cov2 [1,2] patient [3], playing a key role in immune suppression [4]. The same immune suppressive proteins from the same bacteria (elongation factor Tu) was also observed in low amounts in two other patients [5].

Prevotella is known to cause ground glass opacity [6] being used to identify SARS-Cov2 patients [7]. It is also known to increase IL-6 in plasma [8-10], which is correlated with severity/fatalities [11]. In 2003 SARSCov1 outbreak, secondary infection and IL-6 was highly correlated with hospitalization and deaths [12].

\section{Metagenomic presence - known bacteria, and many (10\% of reads) unknown species}

Here, sequencing data (Accid:SRR11347377, Reads=2.64million, Nanopore) from San Diego show Prevotella, Haemophilus parainfluenzae (which can have the same ground glass in CT [13]) and other bacteria in small amounts (SI/bacteria.fa, $\mathrm{N}=23$ ). More intriguingly, there are significant amount of reads with no known matches (SI/nomatches.fa, $\mathrm{N}=200 \mathrm{~K}$, about $10 \%$ ).

\section{Chimeric reads (10\% of reads) involving SARS-Cov2}

Like previous, data $(\mathrm{N}=3)$ showing SARS-Cov2 integration in bacteria [14], there are a significant percentage of chimeric reads (SI/integrated.fa, $\mathrm{N}=250 \mathrm{~K}, \sim 10 \%$ ) having both SARS-Cov2 and something else (sometimes known bacterial reads). Is this sequencing artifact - or is the virus infecting both bacterial and human cells (which would be a first) [15]? It would explain the long incubations and false negatives.

\section{References}

1. Perlman S (2020). Another decade, another coronavirus.

2. Zhu N, Zhang D, Wang W, Li X, Yang B, et al. (2020) A novel coronavirus from patients with pneumonia in China, 2019. New England Journal of Medicine .

Table 1: Bacterial species with number of reads: Prevotellamassilia is a new bacterial species isolated from the human gut [16].

\begin{tabular}{|c|c|}
\hline Species & Number of reads \\
\hline Prevotella & 7 \\
Haemophilus & 7 \\
Prevotellamassilia & 2 \\
Veillonella & 3 \\
\hline
\end{tabular}


3. Wu F, Zhao S, Yu B, Chen Y, Wang W, et al. (2020) Complete genome characterisation of a novel coronavirus associated with severe human respiratory disease in Wuhan. China bioRxiv 24.

4. Chakraborty S (2020). The 2019 Wuhan outbreak could be caused by the bacteria Prevotella, which is aided by the coronavirus, possibly to adhere to epithelial cells - prevotella is present in huge aemounts in patients from both China and Hong Kong. doi:10.31219/osf.io/usztn. URL osf .io/usztn.

5. Chen L, Liu W, Zhang Q, Xu K, Ye G, et al. (2020) RNA based mNGS approach identifies a novel human coronavirus from two individual pneumonia cases in 2019 Wuhan outbreak. Emerging Microbes \& Infections 9: 313-319.

6. Berardino ADM, Inchingolo R, Smargiassi A, Re A, Torelli R, et al. (2014) Empyema caused by prevotella bivia complicating an unusual case of spontaneous chylothorax. Journal of clinical microbiology 52: $1284-1286$.

7. Shi H, Han X, Jiang N, Cao Y, Alwalid O, et al. (2020) Radiological findings from 81 patients with covid-19 pneumonia in wuhan, china: a descriptive study. The Lancet Infectious Diseases .

8. Leite AZ, Rodrigues NdC, Gonzaga MI, Paiolo JCC, de Souza CA, et al. (2017) Detection of increased plasma interleukin-6 levels and prevalence of prevotella copri and bacteroides vulgatus in the feces of type 2 diabetes patients. Frontiers in immunology 8: 1107.

9. Larsen JM (2017) The immune response to prevotella bacteria in chronic inflammatory disease. Immunology 151: 363-374.

10. Choi EY, Jin JY, Choi JI, Choi IS, Kim SJ (2014) Effect of azithromycin on prevotella intermedia lipopolysaccharide-induced production of interleukin- 6 in murine macrophages. European journal of pharmacology 729: 10-16.

11. Chen X, Zhao B, Qu Y, Chen Y, Xiong J, et al. (2020) Detectable serum sars-cov-2 viral load (rnaaemia) is closely associated with drastically elevated interleukin 6 (il-6) level in critically ill covid19 patients. medRxiv .

12. Jiang Y, Xu J, Zhou C, Wu Z, Zhong S, et al. (2005) Characterization of cytokine/chemokine profiles of severe acute respiratory syndrome. American journal of respiratory and critical care medicine 171: $850-857$.

13. Okada F, Ando Y, Tanoue S, Ishii R, Matsushita S, et al. (2012) Radiological findings in acute haemophilus influenzae pulmonary infection. The British journal of radiology 85: 121-126.

14. Chakraborty S (2020). Sequencing data $(n=3)$ shows wuhan coronavirus integration in bacteria (Prevotella mostly). sequencing artifact - or is the virus infecting both bacterial and human cells? doi: 10.31219/osf.io/ktngw. URL osf .io/ktngw.

15. Xu J, Xiang Y (2017) Membrane penetration by bacterial viruses. Journal of virology 91: e00162-17.

16. Ndongo S, Lagier JC, Fournier PE, Raoult D, Khelaifia S (2016) "prevotellamassilia timonensis," a new bacterial species isolated from the human gut. New microbes and new infections 13: 102. 